\title{
THz response of nonequilibrium electrons of highly doped graphene on a polar substrate
}

\author{
Kukhtaruk S. M. \\ Institute of Semiconductor Physics of NASU, 41 Nauky Ave., 03650 Kyiv, Ukraine, \\ e-mail:kukhtaruk@gmail.com
}

Received: 01.11 .2012

\begin{abstract}
We consider high-frequency response of a system consisting of drifting electrons in a highly doped graphene and surface polar optical phonons of a polar substrate. We obtain the dielectric function, the frequencies and the decrement/increment of cooperative plasmon-optical phonon oscillations for this interacting system. We find that the response depends significantly on the degree of nonequilibrium for the electrons. In particular, the interaction between drifting plasmons and surface polar optical phonons leads to instability of the electron subsystem due to Vavilov-Cherenkov effect. We suggest that the hybrid system, a graphene on a polar substrate, is capable of using in amplifiers or generators of $\mathrm{THz}$ electromagnetic radiation.
\end{abstract}

Keywords: graphene, plasmon, optical phonon, instability, dielectric function

PACS: 72.80.Vp, 73.20.Mf, 71.38.-k, 71.45.Gm, 77.22.Ch

UDC: $535.58,535.56,537.5$

\section{Introduction}

As is well known [1], graphene shows a number of remarkable physical properties. In particular, the kinetic energy $E_{k}$ of free carriers as a function of momentum module $|\mathbf{p}|=p$ is linear in a wide energy range [2]:

$$
E_{k}= \pm v_{F} p,
$$

where $v_{F} \approx 10^{8} \mathrm{~cm} / \mathrm{s}$ is the Fermi velocity (the signs ' + ' and '-' in Eq. (1) correspond to electrons and holes, respectively). This symmetry of energy bands allows considering the graphene as a bipolar semiconductor. To work only with electrons, one should have the concentration of electrons much greater than that of holes. This is can be achieved by doping the graphene. It can be doped chemically or by applying some positive electric voltage to a gate, which is generally placed under a substrate. In addition, one can use a so-called electrolytic gate [3] in order to achieve very high electron concentrations (up to $4 \times 10^{14} \mathrm{~cm}^{-2}$ ).

The influence of substrate on the properties of electrons in graphene has been studied in the works [4-10], including the case of nonequilibrium electrons. However, optical properties and cooperative plasma oscillations in highly doped graphene, as well as surface polar optical phonons (SPOP) arising from a polar substrate have been studied only for the equilibrium electrons [1115]. In the present study, we calculate dielectric function of the system mentioned above for the case of nonequilibrium electrons. Basing on this dielectric function, we study oscillations of the coupled system. Although similar studies have been reported earlier [16, 17], they refer to materials where free electrons have a parabolic energy spectrum. 


\section{Basic equation and model criteria}

At first, let us choose a coordinate system and consider an electronic subsystem. Let $O z$ axis be perpendicular to the plane of graphene sheet. Then axes $O x$ and $O y$ would belong to the plane of graphene. The graphene sheet is situated on a polar substrate at $z=0$. The electronic subsystem can be described by a kinetic equation for distribution function $F(\mathbf{r}, \mathbf{p}, t)$ of electrons in highly doped graphene:

$$
\frac{\partial F}{\partial t}+v_{F} \frac{\mathbf{p}}{p} \frac{\partial F}{\partial \mathbf{r}}-\left.e \mathbf{E}\right|_{z=0} \frac{\partial F}{\partial \mathbf{p}}=I\{F\} .
$$

Here $\mathbf{r}=(x, y)$ and $\mathbf{p}=\left(p_{x}, p_{y}\right)$ are respectively the coordinates and the momenta, $-e$ is the electron charge $(e>0), t$ the time, and $I\{F\}$ the collision integral. The total electric field $\mathbf{E}(\mathbf{r}, z, t)=\mathbf{E}_{0}+\mathbf{E}_{s}(\mathbf{r}, z, t)$ consists of an external stationary homogeneous driving electric field $\mathbf{E}_{0}$ and a self-consistent field $\mathbf{E}_{s}(\mathbf{r}, z, t)$ of electrons. The total field in Eq. (2) should be calculated in the plane of graphene, i.e. at $z=0$.

In the case of highly doped graphene, the electron concentration is high and the main mechanism of collision corresponds to electron-electron collisions $[5,18,19]$. Then the stationary spatially uniform solution of Eq. (2) is a shifted Fermi-Dirac distribution function:

$$
f_{0}(\mathbf{p})=\left(\exp \left(\frac{v_{F} p-v_{0} p_{x}-\mu}{k_{B} T}\right)+1\right)^{-1},
$$

where the drift velocity $\mathbf{v}_{0}=\left(v_{0}, 0\right)$ is directed along the $O x$ axis. Here $k_{B}$ and $T$ are respectively the Boltzmann constant and the electron temperature. The chemical potential $\mu$ can be determined from the normalisation condition

$$
\frac{g_{s} g_{v}}{(2 \pi \hbar)^{2}} \int d^{2} p f_{0}=n_{0},
$$

where $g_{s}=2$ and $g_{v}=2$ imply respectively the spin and valley degenerations, $\hbar$ is the reduced Planck constant, and $n_{0}$ the equilibrium surface concentration of electrons.

To describe the electron plasma oscillations, we will use a linear approximation $F(\mathbf{r}, \mathbf{p}, t)=f_{0}(\mathbf{p})+f_{1}(\mathbf{r}, \mathbf{p}, t)$, where $f_{1}(\mathbf{r}, \mathbf{p}, t)$ represents a small perturbation to a function $f_{0}(\mathbf{p})$. We will neglect the collision integral in the linearised kinetic equation for $f_{1}(\mathbf{r}, \mathbf{p}, t)$ :

$$
\frac{\partial f_{1}}{\partial t}+v_{F} \frac{\mathbf{p}}{p} \frac{\partial f_{1}}{\partial \mathbf{r}}-e \mathbf{E}_{0} \frac{\partial f_{1}}{\partial \mathbf{p}}=\left.e \mathbf{E}_{s}\right|_{z=0} \frac{\partial f_{0}}{\partial \mathbf{p}} .
$$

For convenience we will use the scalar potential $\varphi(\mathbf{r}, z, t)$ instead of the field $\mathbf{E}_{s}(\mathbf{r}, z, t)$, which are linked by a simple relation $\mathbf{E}_{s}=-\nabla \varphi$.

Since Eq. (5) does not contain the coordinates and the time explicitly, the quantities $f_{1}(\mathbf{r}, \mathbf{p}, t) \quad$ and $\varphi(\mathbf{r}, z, t) \quad$ can $\quad$ be represented $\quad$ as $\quad f_{1}(\mathbf{r}, \mathbf{p}, t)=f_{\omega, \mathbf{k}}(\mathbf{p}) e^{i \mathbf{k r}-i \omega t+i \cdot 0} \quad$ and $\varphi(\mathbf{r}, z, t)=\varphi_{\omega, \mathbf{k}}(z) e^{i \mathbf{k r}-i \omega t+i \cdot 0}$, where $\omega$ and $\mathbf{k}$ are the frequency and the wave vector of the perturbation, respectively. The infinitesimal term $i \cdot 0$ arises from the principle of causality. It gives the Landau rule to bypass the poles in the integrals (see below). The same rule applies if we add the infinitesimal collision integral $f_{1} / \tau$ at $\tau \rightarrow \infty$ to the $r$. h. s. of the kinetic equation (5). Ukr. J. Phys. Opt. 2013, Volume 14, Issue 1 
the infinitesimal collision integral $f_{1} / \tau$ at $\tau \rightarrow \infty$ to the r. h. s. of the kinetic equation (5). Nevertheless, one should keep in mind that consideration of the case of a finite $\tau$ value does not make sense, since such a collision integral would violate the charge conservation law.

A neglect of the collision integral is valid if

$$
\omega \tau_{p}>>1
$$

where $\tau_{p}$ is the momentum relaxation time of electrons. Use of a classical kinetic equation for the electrons imposes the following restriction on the wave vector value:

$$
k<k_{F},
$$

where $k_{F}=\sqrt{\pi n_{0}}$ is the absolute value of the equilibrium Fermi wave vector. If doping of the graphene is carried out by applying a positive voltage to the gate located under the substrate, our model would be valid if $k d>>1$, where $d$ is the substrate thickness, i.e. the distance between the gate and the graphene sheet.

We will also neglect the term $-e \mathbf{E}_{0} \frac{\partial f_{1}}{\partial \mathbf{p}}$ in Eq. (5). This is valid if the changes in the electron momentum occurring in the field $\mathbf{E}_{0}$ over the time period of the wave are much smaller than the average electron momentum, and if the changes in the electron energy imposed by this field over the spatial period of the wave are much smaller than the average electron energy (i.e., the Fermi energy).

As follows from Eqs. (3) and (4), the chemical potential decreases with increasing drift velocity, so that we do not consider the drift velocity of electrons close to $v_{F}$. If the speeds typical for the electrons are much smaller than the speed of light, Eq. (5) should be solved together with the Poisson equation for the Fourier components of the potential $\varphi_{\omega, \mathbf{k}}(z)$ and the necessary boundary conditions,

$$
\left\{\begin{array}{l}
\frac{d^{2} \varphi_{\omega, \mathbf{k}}}{d z^{2}}-k^{2} \varphi_{\omega, \mathbf{k}}=0 \\
\left.\varphi_{\omega, \mathbf{k}}\right|_{z \rightarrow \pm \infty} \rightarrow 0 \\
\left.\varphi_{\omega, \mathbf{k}}\right|_{z=-\delta}=\left.\varphi_{\omega, \mathbf{k}}\right|_{z=\delta} \\
\left.\frac{d \varphi_{\omega, \mathbf{k}}}{d z}\right|_{z=\delta}-\left.\varepsilon(\omega) \frac{d \varphi_{\omega, \mathbf{k}}}{d z}\right|_{z=-\delta}=\frac{4 \pi e g_{S} g_{v}}{(2 \pi \hbar)^{2}} \int d^{2} p f_{\omega, \mathbf{k}}(\mathbf{p})
\end{array} .\right.
$$

Here $\delta \rightarrow+0$ and the dielectric permittivity $\varepsilon(\omega)$ involved in the last boundary condition is a function of frequency (see, e.g., [20]):

$$
\varepsilon(\omega)=\varepsilon_{\infty}+\frac{\left(\varepsilon_{0}-\varepsilon_{\infty}\right) \omega_{T O}^{2}}{\omega_{T O}^{2}-\omega^{2}-i \gamma_{T O} \omega},
$$

where $\varepsilon_{0}$ and $\varepsilon_{\infty}$ are respectively the static and high-frequency dielectric constants of the substrate, $\omega_{T O}$ is the frequency of transverse optical phonons, and the parameter $\gamma_{T O}$ describes the phonon damping $\left(\gamma_{T O}<<\omega_{T O}\right)$.

Eqs. (3)-(5) and Eqs. (8)-(9) compose a basic system for the problem under consideration. 


\section{Results and discussion}

Using the above system of equations, one can obtain the dielectric function for the interacting plasmons and substrate phonons:

$$
\varepsilon(\Omega, \mathbf{K})=1+\Omega_{e} \frac{\Omega^{2}-1+i \Gamma_{T O} \Omega}{\Omega^{2}-\Omega_{0}^{2}+i \Gamma_{T O} \Omega} \int \frac{d^{2} P}{\left(\Omega-\frac{\mathbf{K P}}{P}+i \cdot 0\right)} \frac{\mathbf{K}}{K} \frac{\partial f_{0}}{\partial \mathbf{P}},
$$

where for convenience the following dimensionless variables are introduced: $\Omega=\frac{\omega}{\omega_{T O}}$, $K=\frac{k v_{F}}{\omega_{T O}}=\sqrt{K_{x}^{2}+K_{y}^{2}}, \quad \Omega_{e}=\frac{4 \pi e^{2} g_{S} g_{v} k_{B} T}{(2 \pi \hbar)^{2} v_{F} \omega_{T O}\left(1+\varepsilon_{\infty}\right)}, \quad \Omega_{0}=\frac{\omega_{S O}}{\omega_{T O}}, \quad \Gamma_{T O}=\frac{\gamma_{T O}}{\omega_{T O}}$, and $V_{0}=\frac{v_{0}}{v_{F}}$.

Here $\omega_{S O}=\sqrt{\frac{1+\varepsilon_{0}}{1+\varepsilon_{\infty}}} \omega_{T O}$ denotes the frequency of surface optical phonons [13]. Notice that the dielectric function given by Eq. (10) is anisotropic if the electron drift is present. This could lead to rotation of the light polarisation plane. Also, if we put $\omega_{S O}=\omega_{T O}$ or $\varepsilon_{0}=\varepsilon_{\infty}$, we obtain the dielectric function for the pure plasma oscillations in highly doped graphene, because the width of the residual radiation band is zero in this case.

It is well-known that the Landau collisionless damping is absent in highly doped graphene plasma whenever $k<<k_{F}$. This is because the pure plasmon branch does not get to the region where the imaginary part of the dielectric function is nonzero. As shown for the equilibrium case [12], the interaction of plasmons with the SPOP leads to splitting of the pure plasmon branch into two branches, $\Omega_{+}$and $\Omega_{-}$. The damping decrement $\Gamma_{+}$corresponding to $\Omega_{+}$is equal to $-\Gamma_{T O} / 2$, since this branch passes through the region where the imaginary part of the integral appearing in Eq. (10) is zero. From the other hand, the branch $\Omega_{-}$does pass through the region where the imaginary part of the integral in Eq. (10) is nonzero. In this region both the term $\Gamma_{T O}$ and the imaginary part of the integral involved in Eq. (10) give contributions to the $\Gamma_{-}$parameter. Thus, the nature of damping of the cooperative plasma and the SPOP oscillations for the $\Omega_{-}$ branch is the same as the nature of the Landau collisionless damping. As will be shown, the similar situation also occurs for the nonequilibrium case. However, starting from some values of the drift velocity and the $\mathbf{K}$ parameter, the damping effect is replaced by growing oscillations that correspond to electrical instability of our system.

When performing numerical calculations, we have chosen $\mathrm{TlCl}$ as a substrate material. This material $[21,22]$ has a very wide residual radiation band, since $\varepsilon_{0} \approx 37.2$ and $\varepsilon_{\infty} \approx 5$ at the helium temperature. Moreover, we have $\omega_{T O} \approx 1.15 \times 10^{13} \mathrm{~s}^{-1}$ and $\Gamma_{T O} \approx 0.066$ for TlCl. We take the electron concentration $n_{0}=10^{13} \mathrm{~cm}^{-2}$ and the electron temperature $T=200 \mathrm{~K}$ for our estimations. For the parameters chosen as above, the dimensionless equilibrium Fermi wave vector is equal to $K_{F}=\frac{v_{F} k_{F}}{\omega_{T O}} \approx 48.7$. The chemical potentials for the cases of $V_{0}=0$ and $V_{0}=0.4$ are $\mu \approx 50 \mathrm{meV}$ and $\mu \approx 41.6 \mathrm{meV}$, so that we obtain $\frac{\mu}{k_{B} T}=2.9$ and $\frac{\mu}{k_{B} T}=2.4$, respectively. For 
simplicity, we put $K_{y}=0$. Further on, we will present the results for the values of parameters that satisfy the inequalities (6) and (7), and the other criteria of our model.
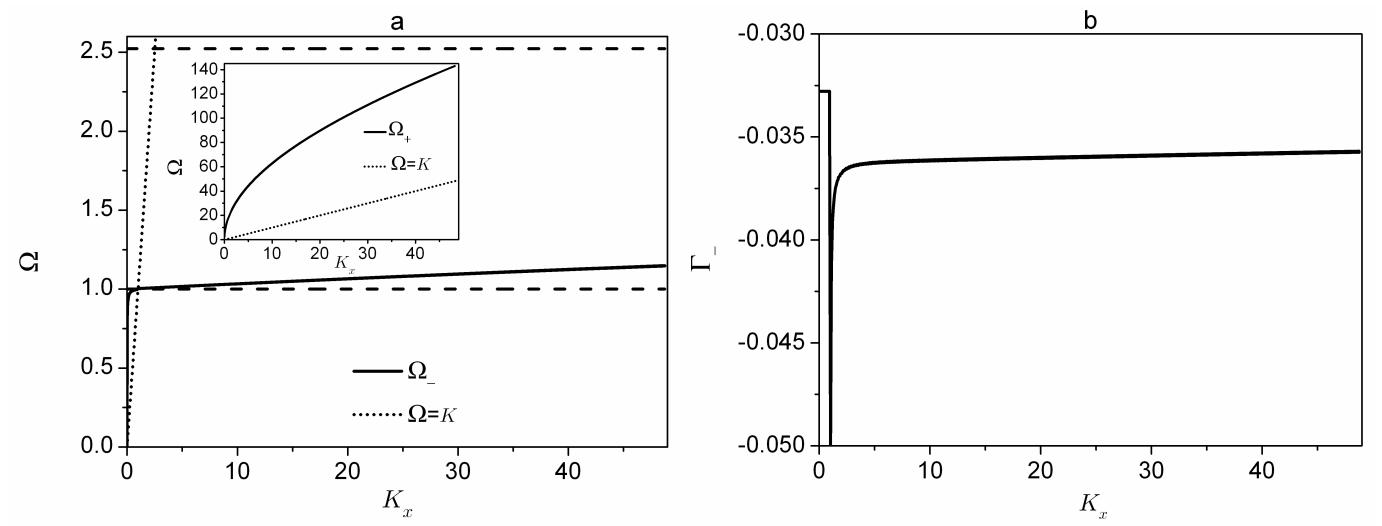

Fig. 1. (a) Frequency branch $\Omega_{-}\left(K_{x}\right)$ at $V_{0}=0$. Dash line restricts the residual radiation band. Branch $\Omega_{+}\left(K_{x}\right)$ is presented in the inset. (b) Decrement $\Gamma_{-}$as a function of $K_{x}$.

As seen from Fig. 1a, the frequency branch $\Omega_{-}$passes close to $\Omega=1$ and, due to a strong interaction, this branch never reaches the value $\Omega=\Omega_{0} \approx 2.5$ corresponding to $\omega_{S O}$, even at $K_{x}=K_{F}$. Since the branch $\Omega_{-}$crosses the line $|\Omega|=K$, the damping caused only by the phonon damping corresponds to this branch in the region $\Omega>K$ (i.e., $\Gamma_{-}=-\Gamma_{T O} / 2$ ). Therefore the total damping caused by the phonon damping and the Landau damping mechanisms corresponds to this branch in the region $|\Omega|<K$. A cutting behaviour of $\Gamma_{-}\left(K_{x}\right)$ can be explained by the fact that the imaginary part appearing in Eq. (10) has a point of discontinuity of the second kind at $|\Omega|=K$.

One should also notice a very rapid growth of the $\Omega_{+}$and $\Omega_{-}$branches at small $K_{x}$. Such behaviour of the frequency branches leads to high group velocity of the wave in the region of the rapid growth. As is well known, the group velocity is related to energy transfer. Under electric field applied, a large part of the energy is given to almost dispersionless SPOP, of which group velocity is very small. As a result, the heat is not rejecting from the graphene. In the case of coupled vibrations of graphene plasmons and the SPOP, the group velocity of the waves corresponding to these vibrations is significantly greater. Hence, these waves could provide heat rejection from the graphene.

The both frequency branches for the nonequilibrium case (see Fig. 2) differ not very much from those shown in Fig. 1. From the other hand, the parameter $\Gamma_{-}$changes its sign, which corresponds to electrical instability of the system. In the absence of phonon damping, the $\Gamma_{-}$sign reverses strictly at $\Omega=V_{0} K_{x}$, which corresponds to the Vavilov-Cherenkov effect. However, the total function $\Gamma_{-}$shifts by a half of the phonon damping parameter $\Gamma_{T O}$ if $\Gamma_{T O}$ remains nonzero (as in Fig. 2). It is worthwhile to notice that the effect of instability can be used for generating or amplifying the $\mathrm{THz}$ electromagnetic radiation.

When the electron drift is present, the electron temperature can be significantly higher than the ambient temperature. Our analysis shows that the effect of instability can also occur in the case of significant heating, whenever one can create high enough electron concentrations. 

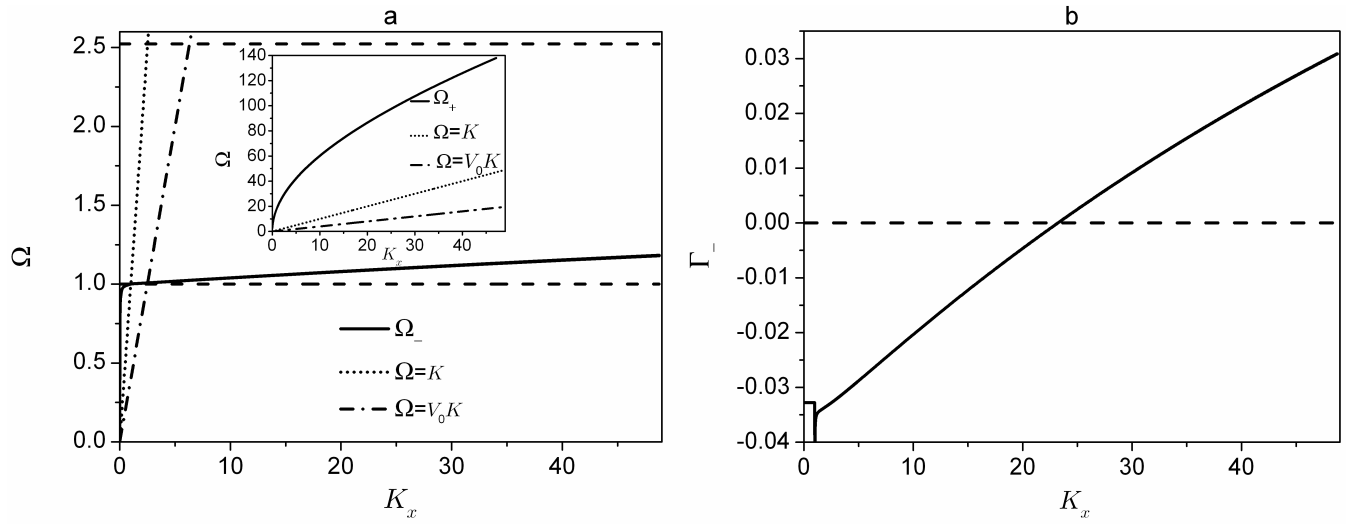

Fig. 2. The same dependences as shown in Fig. 1 calculated at $V_{0}=0.4$.

In conclusion, we have theoretically studied a hybrid system composed of a highly doped graphene and a polar substrate. We have found the $\mathrm{THz}$ response of the nonequilibrium electrons interacting with the SPOP. The response depends significantly on the electron drift velocity. In particular, the damping of oscillations of the system can give way to their growth, if the drift velocity is large. We suggest that the hybrid system under consideration is capable of using for amplification or generation of the $\mathrm{THz}$ electromagnetic radiation.

\section{Acknowledgement}

The author is grateful to Prof. V. A. Kochelap for valuable suggestions and permanent attention to this work and to Prof. M. V. Strikha for valuable discussions on the subject. This work is partially supported by the State Fundamental Research Fund of Ukraine.

\section{References}

1. Castro Neto A H, Guinea F, Peres N M R, Novoselov K S and Geim A K, 2009. The electronic properties of graphene. Rev. Mod. Phys. 81: 109-162.

2. Wallace P R, 1947. The band theory of graphite. Phys. Rev. 71: 622-634.

3. Efetov D K and Kim P, 2010. Controlling electron-phonon interactions in fraphene at ultrahigh carrier densities. Phys. Rev. Lett. 105: 256805.

4. Fratini S and Guinea F, 2008. Substrate-limited electron dynamics in graphene. Phys. Rev. B. 77: 195415.

5. Li X, Barry E A, Zavada J M, Buongiorno Nardelli M and Kim K W, 2010. Surface polar phonon dominated electron transport in graphene. Appl. Phys. Lett. 97: 232105.

6. DaSilva A M, Zou K, Jain J K and Zhu J, 2010. Mechanism for current saturation and energy dissipation in graphene transistors. Phys. Rev. Lett. 104: 236601.

7. Perebeinos V and Avouris $P, 2010$. Inelastic scattering and current saturation in graphene. Phys. Rev. B. 81: 195442.

8. Zou K, Hong X, Keefer D and Zhu J, 2010. Deposition of high-quality $\mathrm{HfO}_{2}$ on graphene and the effect of remote oxide phonon scattering. Phys. Rev. Lett. 105: 126601.

9. Konar A, Fang T and Jena D, 2010. Effect of high- $\kappa$ gate dielectrics on charge transport in graphene-based field effect transistors. Phys. Rev. B. 82: 115452.

10. Strikha M V, 2012. Non-volatile memory and IR radiation modulators based upon grapheneon-ferroelectric substrate. A review. Ukr. J. Phys. Opt., Suppl. 3. Sci. Horiz. 13: S5-S26.

11. Jablan M, Buljan H and Soljačić M, 2009. Plasmonics in graphene at infrared frequencies. Phys. Rev. B. 80: 245435. 
12. Hwang E H, Sensarma R and Das Sarma S, 2010. Plasmon-phonon coupling in graphene. Phys. Rev. B. 82: 195406.

13. Koch R J, Seyller Th and Schaefer J A, 2010. Strong phonon-plasmon coupled modes in the graphene/silicon carbide heterosystem. Phys. Rev. B. 82: 201413(R).

14. Jablan M, Soljačić M and Buljan H, 2011. Unconventional plasmon-phonon coupling in graphene. Phys. Rev. B. 83: 161409(R).

15. Fei Z, Andreev G O, Bao W, Zhang L M, McLeod A S, Wang C, Stewart M K, Zhao Z, Dominguez G, Thiemens M, Fogler M M, Tauber M J, Castro-Neto A H, Ning Lau C, Keilmann F and Basov D N, 2011. Infrared nanoscopy of dirac plasmons at the graphene- $\mathrm{SiO}_{2}$ interface. Nano Lett. 11: 4701-5.

16. Kukhtaruk S M, 2008. High-frequency properties of systems with drifting electrons and polar optical phonons. Semicond. Phys., Quant. Electron. and Optoelectron. 11: 43-49.

17. Sydoruk O, Kalinin V and Solymar L, 2010. Terahertz instability of optical phonons interacting with plasmons in two-dimensional electron channels. Appl. Phys. Lett. 97: 062107.

18. Li X, Barry E A, Zavada J M, Buongiorno Nardelli M and Kim K W, 2010. Influence of electron-electron scattering on transport characteristics in monolayer graphene. Appl. Phys. Lett. 97: 082101.

19. Fang T, Konar A, Xing H and Jena D, 2011. High-field transport in two-dimensional graphene. Phys. Rev. B. 84: 125450.

20. Born M and Huang K. Dynamical theory of crystal lattices. Oxford: Clarendon Press (1954).

21. Lowndes R P, 1972. Anharmonicity in the silver and thallium halides: far-infrared dielectric response. Phys. Rev. B. 6: 1490-1498.

22. Lowndes R P, 1972. Anharmonicity in the silver and thallium halides: low-frequency dielectric response. Phys. Rev. B. 6: 4667-4674.

Kukhtaruk S. M., 2013. THz response of nonequilibrium electrons of highly doped graphene on a polar substrate. Ukr.J.Phys.Opt. 14: $24-30$.

Анотація. Розглянуто надвисокочастотний відгук системи дрейфуючих електронів сильно легованого графену та поверхневих оптичних фононів полярної підкладки. Для взаємодіючої системи розраховано діелектричну функиію, частоти та декремент/інкремент спільних коливань. Показано, щзо відгук системи суттєво залежить від ступеня нерівноважності електронів. Зокрема, взаємодія між плазмонами та поверхневими оптичними фононами приводить до дрейфової нестійкості, зумовленої ефектом Вавілова-Черенкова. Розглянуту систему можна використовувати для генерації або підсилення терагериового електромагнітного випромінювання. 